\title{
Acute interstitial pulmonary edema
}

\author{
Julian S Tam MD ${ }^{1}$, Derek A Fladeland MD², Christopher A Hergott MD³, Donald W Cockcroft MD ${ }^{3}$
}

A 68-year-old woman presented to the emergency department of a tertiary care hospital with the chief complaint of hemoptysis. She had been coughing up dark blood in teaspoon-size amounts and experiencing increased dyspnea of an acute onset for the past day. She was in the midst of treatment with moxifloxacin and prednisone for an acute exacerbation of chronic obstructive pulmonary disease.

She had been followed by a respirologist for multiple calcified pulmonary nodules that were stable on imaging. Investigations for her nodules, which included bronchoscopy, serology for histoplasmosis and coccidioidomycosis, and sputum culture and acid-fast bacilli, had been negative to date.

The patient described recurrent episodes of breathlessness during the previous three months that appeared to respond to therapy with antibiotics.

She had a 40 pack-year history of smoking and quit 11 years before presentation. Her history also included chronic obstructive pulmonary disease, hypertension, dyslipidemia and a previous transient ischemic attack. Echocardiography revealed diastolic dysfunction. A physical examination was significant for elevated jugular venous pressure and bilateral crackles on lung auscultation.

A contrast-enhanced computed tomography pulmonary angiogram was ordered in the emergency department. The study was negative for pulmonary embolism, but revealed marked smooth interlobular septal thickening and fissural thickening extending to the upper lung zones (Figures 1A and 1B). Bilateral small pleural effusions were visualized. Her pulmonary nodules were unchanged. Findings from the study were suggestive of acute interstitial pulmonary edema.

The patient was admitted to hospital under the care of the cardiology service for management of congestive heart failure and pulmonary edema. Her symptoms improved promptly with diuresis.

She would later be diagnosed with primary amyloidosis; her heart failure was attributed to amyloid cardiomyopathy. The patient also underwent a surgical lung biopsy that led to the diagnosis of vascular and nodular pulmonary amyloidosis. She received chemotherapy for her amyloidosis.
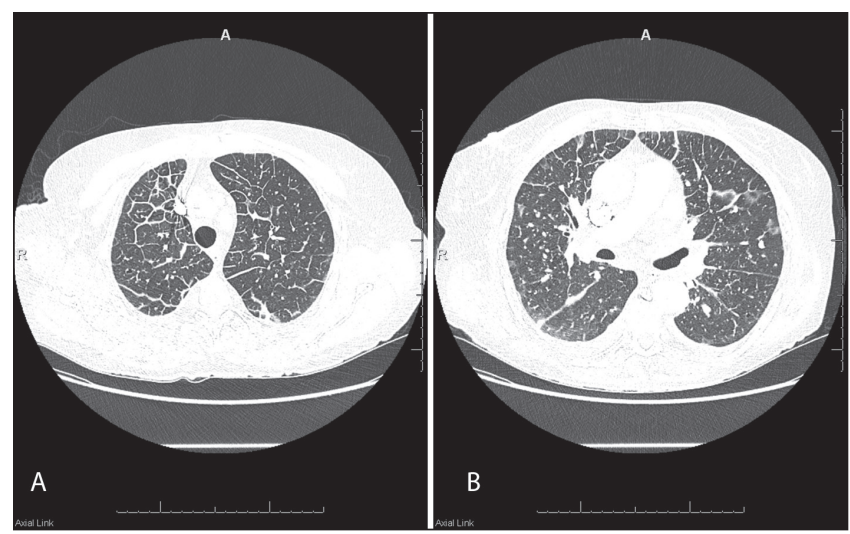

Figures 1) A and B Axial contrast-enhanced computed tomographic pulmonary angiogram on lung windows revealing smooth interlobular septal thickening and stable pulmonary nodules
The patient died of pneumonia in hospital 13 months after her initial admission.

\section{KEY LEARNING POINTS}

- Interlobular septal thickening may be encountered in a variety of conditions. The radiographic finding can be classified into smooth, nodular and irregular forms (1).

- Similar to our patient, smooth interlobular septal thickening may be an imaging manifestation of cardiogenic interstitial pulmonary edema. It may also encountered in lymphangitic carcinomatosis (2).

- Smooth interlobular septal thickening superimposed on groundglass opacities is known as the 'crazy-paving' pattern (Figure 2). Johkoh et al (3) identified 15 diseases associated with this pattern. The finding was most prevalent in alveolar proteinosis, and was also present in diffuse alveolar damage superimposed on usual interstitial pneumonitis, acute interstitial pneumonia, acute respiratory distress syndrome, cardiogenic pulmonary edema, drug-induced pneumonitis, pulmonary hemorrhage, cryptogenic organizing pneumonia, chronic eosinophilic pneumonia and Pneumocystis jirovecii-induced pneumonia (3).

- The differential diagnosis for nodular interlobular septal thickening includes sarcoidosis and lymphangitic carcinomatosis (Figures 3 and 4) (4,5).

- Pulmonary fibrosis can lead to irregular interlobular septal thickening (Figure 5) (6).

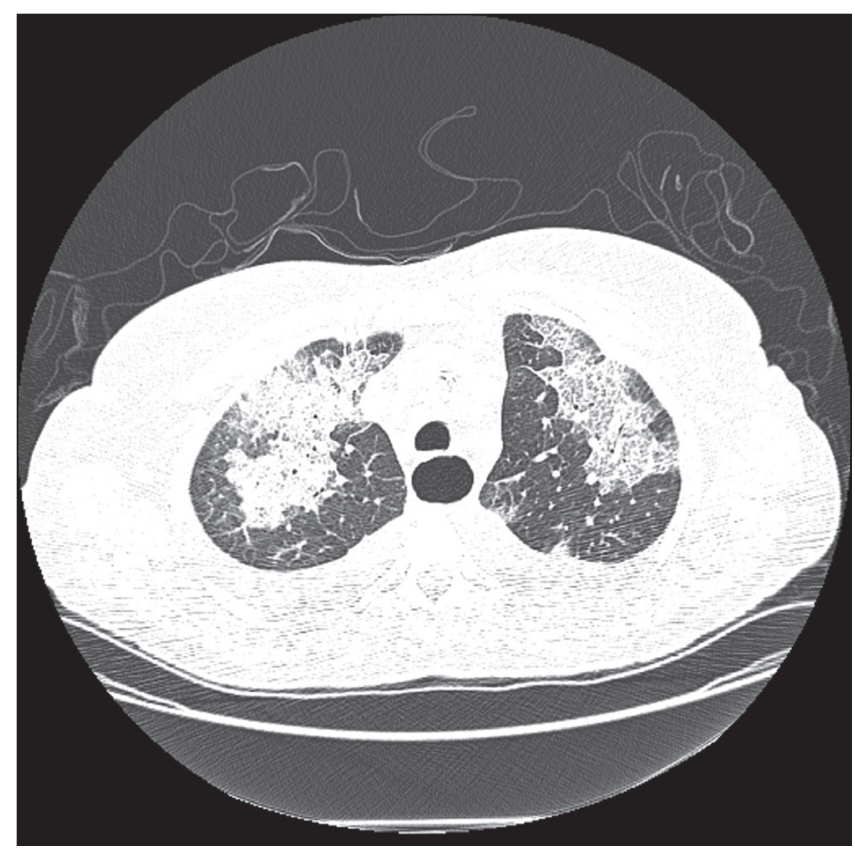

Figure 2) The crazy-paving pattern

${ }^{1}$ Division of Respirology, St Micheal's Hospital, Toronto, Ontario; ${ }^{2}$ Department of Medical Imaging; ${ }^{3}$ Division of Respirology, Critical Care and Sleep Medicine, Department of Medicine; University of Saskatchewan, Saskatoon, Saskatchewan

Correspondence: Dr Julian S Tam, Division of Respirology, St Micheal's Hospital, 30 Bond Street, Room 6-037, Bond Wing, Toronto,

Ontario M5B 1W8. Telephone 416-864-5409, fax 416-864-5651, e-mail contact@juliantam.com 


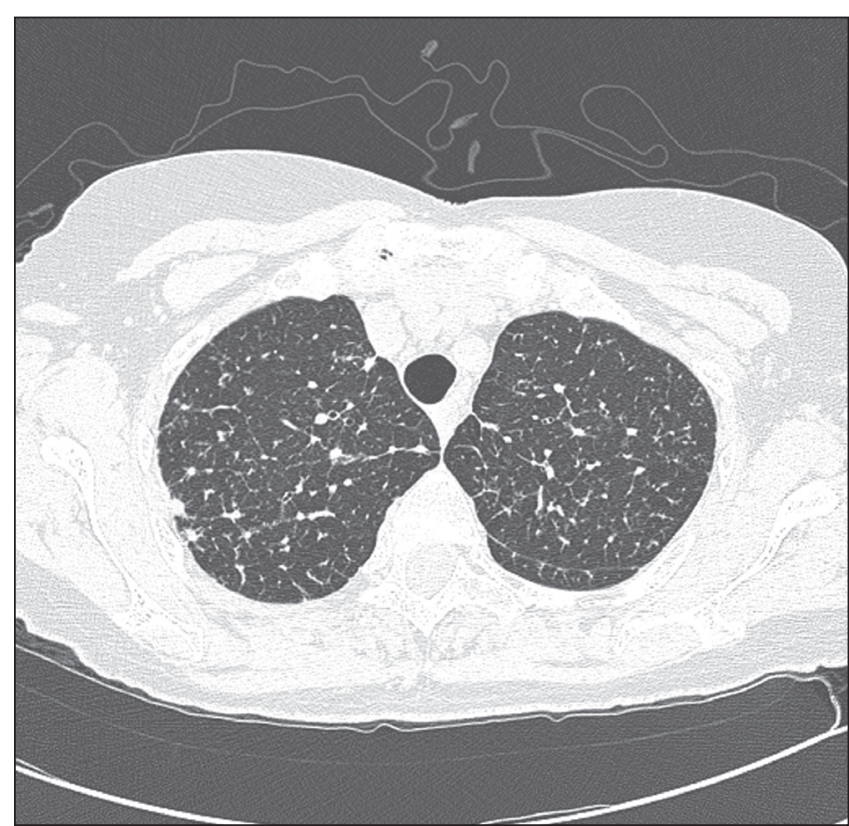

Figure 3) Nodular interlobular septal thickening in a patient with sarcoidosis

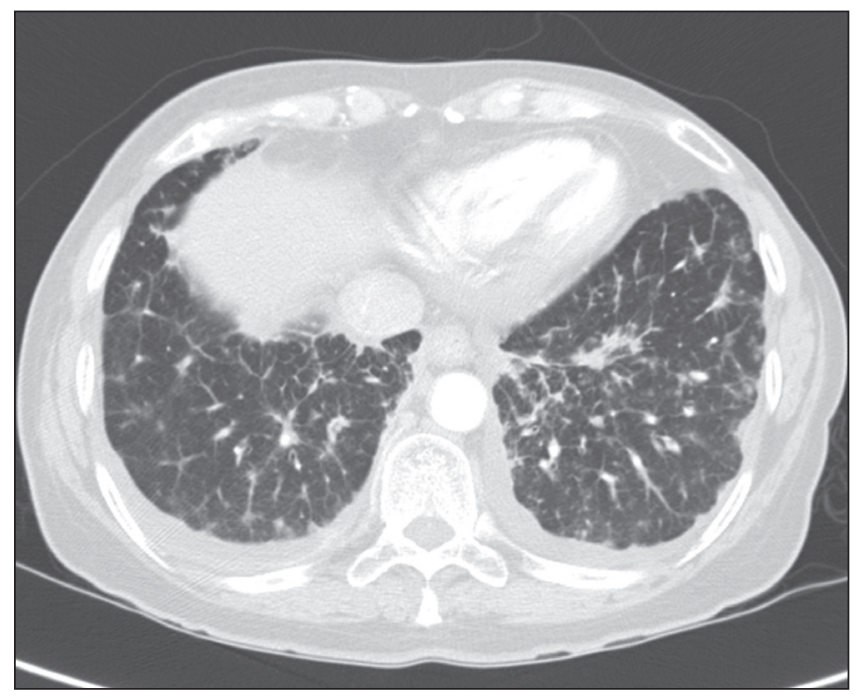

Figure 4) Nodular interlobular septal thickening in a patient with lymphangitic carcinomatosis

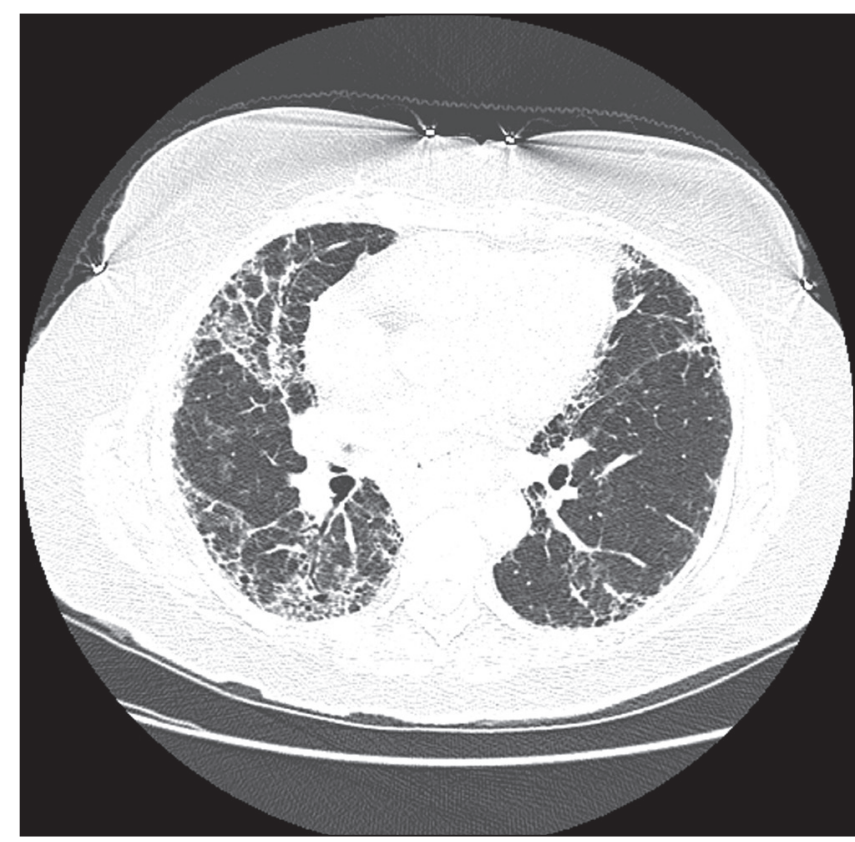

Figure 5) Irregular interlobular septal thickening in a patient with usual interstitial pneumonitis

\section{REFERENCES}

1. Austin JH, Müller NL, Friedman PJ, et al. Glossary of terms for CT of the lungs: Recommendations of the Nomenclature Committee of the Fleischner Society. Radiology 1996;200:327-31.

2. Munk PL, Müller NL, Miller RR, et al. Pulmonary lymphangitic carcinomatosis: CT and pathologic findings. Radiology 1988;166:705-9.

3. Johkoh T, Itoh H, Müller NL, et al. Crazy-paving appearance at thin-section CT: Spectrum of disease and pathologic findings. Radiology 1999;211:155-60.

4. Müller NL, Kullnig P, Miller RR. The CT findings of pulmonary sarcoidosis: Analysis of 25 patients. Am J Roentgenol 1989;152:1179-82

5. Ren H, Hruban RH, Kuhlman JE, et al. Computed tomography of inflation-fixed lungs: The beaded septum sign of pulmonary metastases. J Comp Assist Tomog 1989;13:411-6.

6. Kang EY, Grenier P, Laurent F, et al. Interlobular septal thickening: Patterns at high-resolution computed tomography. J Thorac Imag 1996;11:260-4.

The 'Images in Respiratory Medicine' section of the Canadian Respiratory Journal aims to highlight the importance of visual interpretation, whether physiological, radiological, bronchoscopic, surgical/thorascopic or histological, in the diagnosis of chest diseases. Submissions should exemplify a classic, particularly dramatic or intriguing presentation of a disease while offering an important educational message to the reader (insightful diagnostic pearls or differential diagnosis, etc). This section is not intended to be a vehicle for publication of case reports (see the Clinical-Pathologic-Conferences for case-based leaning series). 


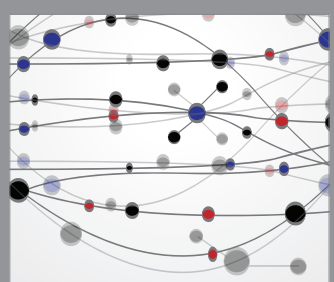

The Scientific World Journal
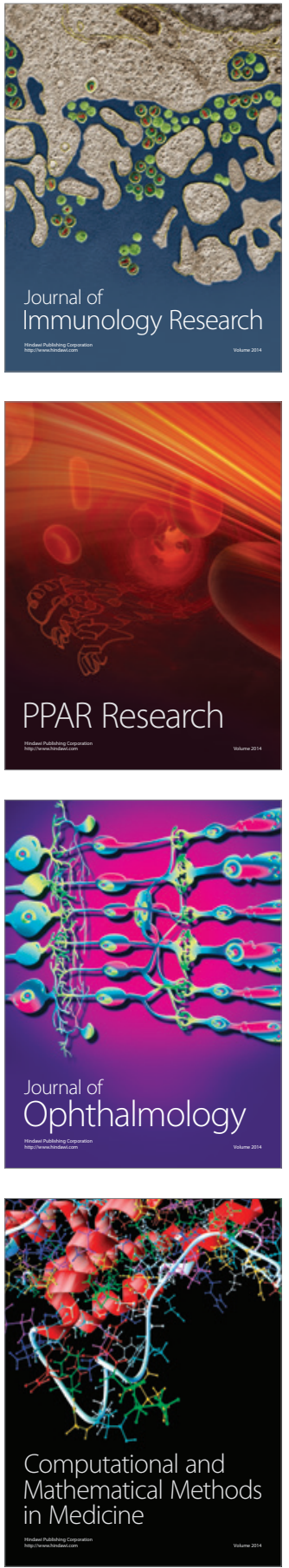

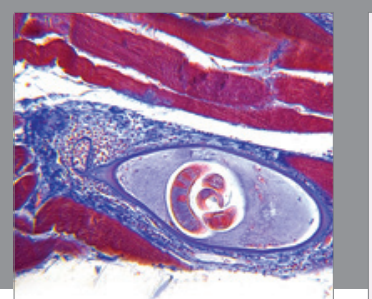

Gastroenterology Research and Practice

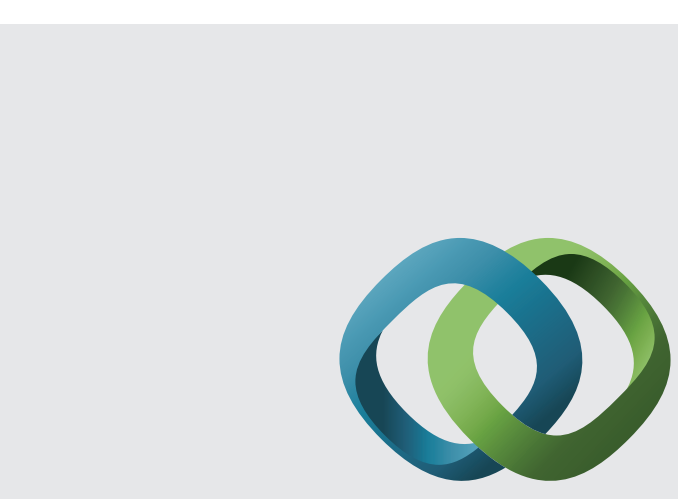

\section{Hindawi}

Submit your manuscripts at

http://www.hindawi.com
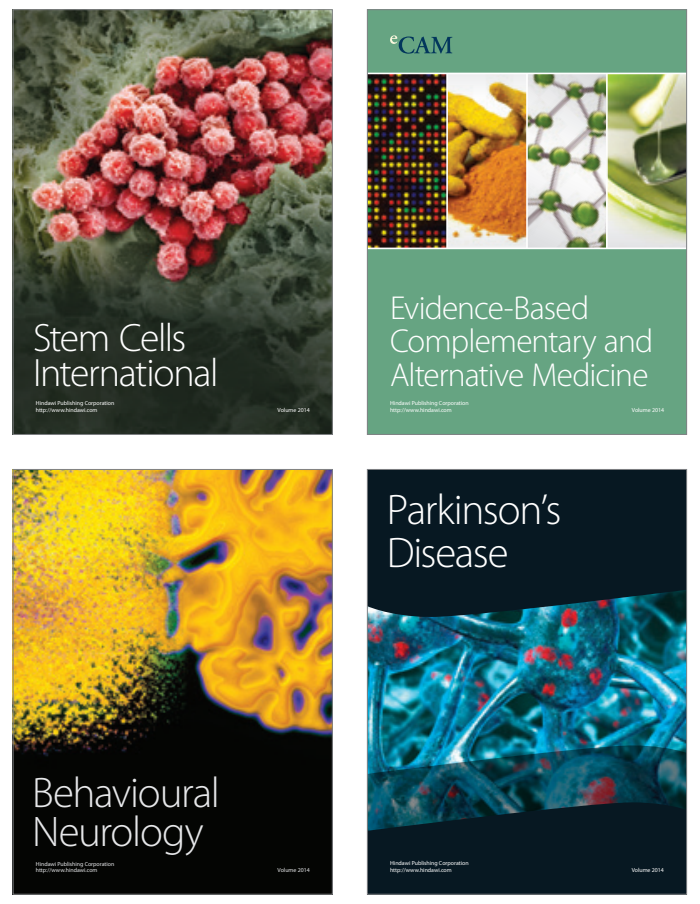
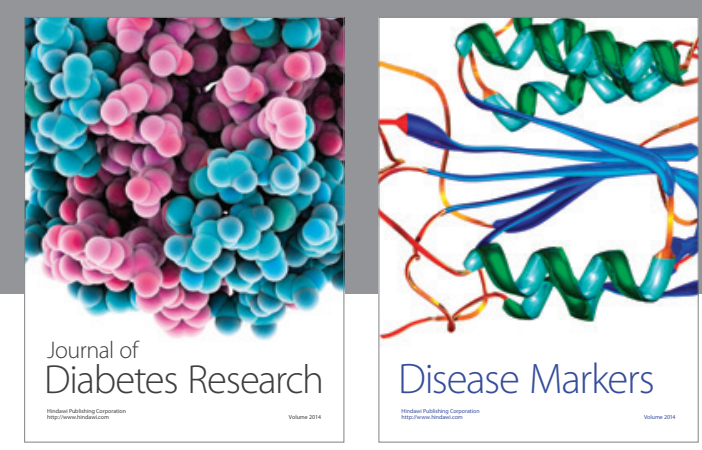

Disease Markers
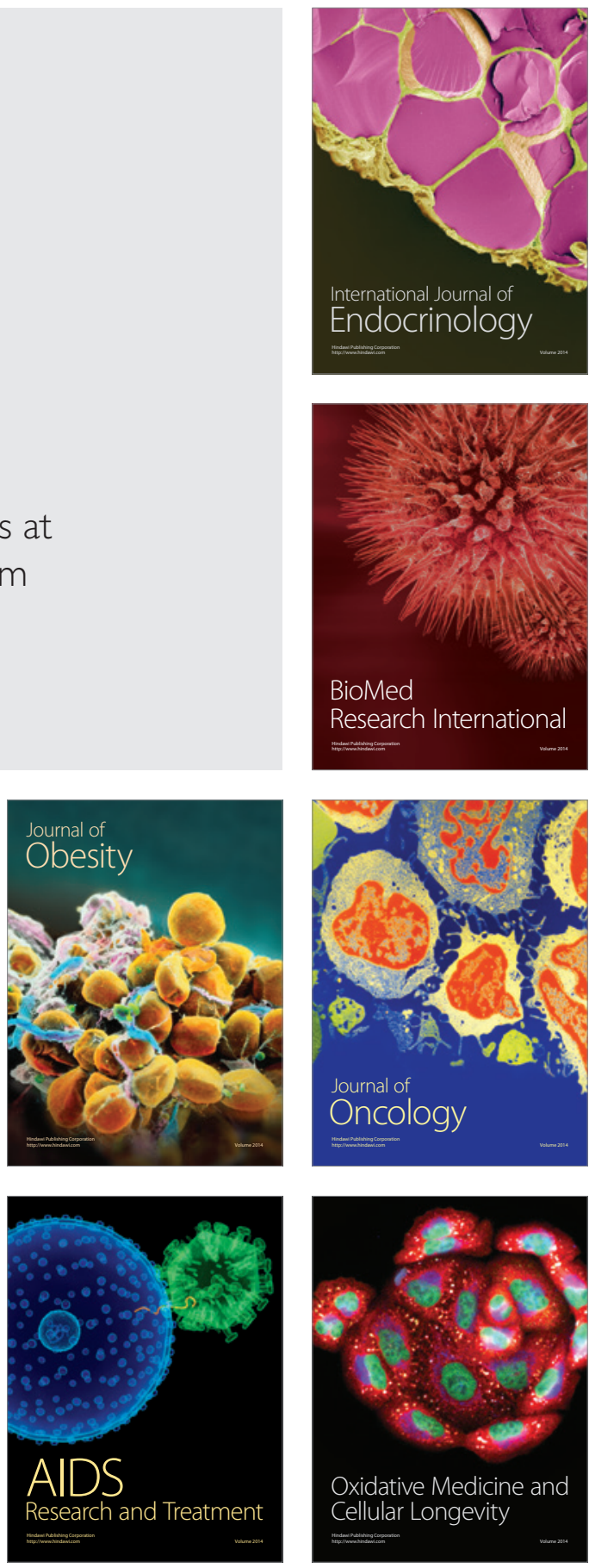